\title{
Alinhamento do plano sagital e resultados funcionais após cirurgia para a cifose de Scheuermann*
}

\section{Sagittal Plane Alignment and Functional Outcomes Following Surgery for Scheuermann Kyphosis}

\author{
Luísa Vital ${ }^{1}$ Bernardo Nunes ${ }^{1}$ Sara Almeida Santos ${ }^{1}$ Vitorino Veludo ${ }^{2}$ Francisco Serdoura ${ }^{2}$ \\ André Pinho ${ }^{3}$ \\ ${ }^{1}$ Departamento de Ortopedia e Traumatologia, Centro Hospitalar \\ Universitário de São João, Porto, Portugal \\ ${ }^{2}$ Centro Hospitalar Universitário de São João, Porto, Portugal \\ ${ }^{3}$ Unidade de Anatomia, Centro Hospitalar Universitário de \\ São João, Porto, Portugal \\ Endereço para correspondência Luísa Vital, Departamento de \\ Ortopedia e Traumatologia, Centro Hospitalar Universitário de São \\ João, Alameda Professor Hernâni Monteiro, Porto, Portugal \\ (e-mail: luisavital1203@gmail.com).
}

Rev Bras Ortop 2021;56(4):446-452.

\section{Resumo \\ Palavras-chave \\ - doença de Scheuermann \\ - coluna vertebral \\ - vértebras torácicas \\ - fusão espinhal \\ - cifose \\ Objetivo Avaliar e correlacionar os parâmetros pélvicos, o equilíbrio sagital (SB), e o resultado funcional dos pacientes submetidos ao tratamento cirúrgico da hipercifose de Scheuermann (SK). \\ Métodos Foram incluídos pacientes submetidos à cirurgia entre janeiro de 2005 e dezembro de 2016. Foram obtidas as seguintes medidas radiográficas: cifose torácica (TK); lordose lombar (LL); SB; incidência pélvica (PI); inclinação pélvica (PT); e inclinação sacral (SS). Complicações foram registradas durante o período de seguimento. \\ Resultados Foram incluídos 19 pacientes ( 16 homens): a cifose pré-operatória média foi de $83^{\circ}$, e, após a cirurgia, de $57^{\circ}$. A LL média pré-operatória foi de $66^{\circ}$, com uma correção espontânea pós-operatória de $47^{\circ}$. Quanto aos parâmetros pélvicos pré- operatórios, as médias de PI, PT e SS foram de $48^{\circ}, 10^{\circ}$ e $39^{\circ}$, respectivamente. No pós- operatório, esses valores foram de $50^{\circ}, 16^{\circ}$ e $35^{\circ}$, respectivamente. O SB pré-operatório foi neutro e mantido após correção cirúrgica. Quanto às complicações durante o período de seguimento, foram observadas três cifoses juncionais - duas necessitando de cirurgia de revisão, uma má consolidação, e uma deiscência de sutura. Em relação aos resultados funcionais, a pontuação média no questionário de 22 itens da Scoliosis Research Society (SRS-22) foi de 4,04, e verificou-se que o SB no pós-operatório não teve influência no desfecho funcional $(p=0,125)$, nem na LL pós-operatória $(p=0,851)$.}

\footnotetext{
Trabalho desenvolvido no Centro Hospitalar Universitário de São João, Porto, Portugal.
}

recebido

26 de Junho de 2020

aceito

16 de Setembro de 2020
DOI https://doi.org/ $10.1055 / \mathrm{s}-0041-1724078$ ISSN 0102-3616.
(C) 2021. Sociedade Brasileira de Ortopedia e Traumatologia. All rights reserved.

This is an open access article published by Thieme under the terms of the Creative Commons Attribution-NonDerivative-NonCommercial-License, permitting copying and reproduction so long as the original work is given appropriate credit. Contents may not be used for commercial purposes, or adapted, remixed, transformed or built upon. (https://creativecommons.org/ licenses/by-nc-nd/4.0/) Thieme Revinter Publicações Ltda., Rua do Matoso 170, Rio de Janeiro, RJ, CEP 20270-135, Brazil 
Conclusão Verificamos uma melhora espontânea da hiperlordose lombar em níveis não incluídos na fusão após a correção da TK. Embora os resultados funcionais pósoperatórios tenham sido globalmente elevados, não encontramos nenhuma relação estatisticamente significativa com a TK nem com a LL. A PI elevada está associada a maior complicação da cifose juncional proximal (PJK), e esses parâmetros pélvicos devem ser considerados no momento do tratamento cirúrgico da cifose de Scheuermann.

\begin{abstract}
Keywords

- Scheuermann disease

- spine

- thoracic vertebrae

- spinal fusion

- kyphosis

Objective To evaluate and correlate the pelvic parameters, the sagittal balance (SB), and the functional outcome of the patients submitted to surgical treatment for Scheuermann hyperkyphosis (SK).

Methods Patients submitted to surgery between January 2005 and December 2016 were included. The following radiographic measurements were obtained: thoracic kyphosis (TK); lumbar lordosis (LL); SB; pelvic incidence (PI); pelvic tilt (PT); and sacral slope (SS). Complications during the follow-up period were recorded.

Results A total of 19 patients were included (16 males): the mean preoperative kyphosis was of $83^{\circ}$, and the postoperative kyphosis was of $57^{\circ}$. The mean preoperative lumbar lordosis was of $66^{\circ}$, with a postoperative spontaneous correction of $47^{\circ}$. Regarding the preoperative pelvic parameters, the average PI, PT and SS were of $48^{\circ}, 10^{\circ}$ and $39^{\circ}$ respectively. In the postoperative period, these values were of $50^{\circ}, 16^{\circ}$ and $35^{\circ}$ respectively. The preoperative SB was neutral, and it was maintained after the surgical correction. Concerning complications during the follow-up period, three junctional kyphosis were observed-two requiring revision surgery, one nonunion, and one dehiscence of the surgical wound. Regarding the functional results, the average score on the Scoliosis Research Society-22 (SRS-22) patient questionnaire was of 4.04, and we verified that the SB obtained in the postoperative period had no influence on the functional outcome $(p=0.125)$ nor on the postoperative LL $(p=0.851)$.

Conclusion We verified a spontaneous improvement in the lumbar hyperlordosis at levels not included in the fusion after correction of the TK. Although the postoperative functional results were globally high, we did not find any statistically significant relationship with TK nor LLs. high PI is associated with a greater rate of complications regarding the proximal junctional kyphosis (PJK), and these pelvic parameters should be considered at the time of the SK surgical treatment.
\end{abstract}

\section{Introdução}

A hipercifose de Scheuermann (SK) é uma deformidade no plano sagital com incidência que varia de $0,4 \%$ a $8 \%$, com uma prevalência masculina ligeiramente maior, e é atualmente a causa mais comum de hipercifose rígida na população pediátrica. $^{1,2}$

O diagnóstico é frequentemente feito em adolescentes que apresentam sintomas crônicos de dor lombar leve e uma deformidade cosmética típica da coluna torácica ou toracolombar. De acordo com Sorensen, ${ }^{3}$ os critérios radiográficos para o diagnóstico incluem $\mathrm{TK}>40^{\circ}$ ou cifose toracolombar (TLK) $>30^{\circ}$, e pelo menos 3 corpos vertebrais consecutivos com um mínimo de $5^{\circ}$ de encunhamento.

O objetivo do tratamento cirúrgico da SK não é apenas a correção da deformidade, como também uma melhora sinto- mática e cosmética. Um grande número de estudos relata desfechos radiográficos, mas a maioria deles se concentra principalmente nos parâmetros da coluna vertebral, sem incluir a avaliação pélvica e sua relação com o equilíbrio sagital (SB) e a qualidade de vida. A incidência pélvica (PI) é uma característica fundamental da pelve definida como uma característica anatômica única para cada indivíduo, que se estabiliza no final do crescimento e determina a posição relativa da placa sacral em relação às cabeças femorais. A inclinação sacral (SS) é o ângulo do plano sacral para o plano horizontal, e determina a posição da coluna lombar, uma vez que o plano sacral forma a base da coluna vertebral. A inclinação pélvica (PT) é definida pelo ângulo entre uma linha vertical ao longo da cabeça femoral e uma linha do plano médio sacral à cabeça femoral, e determina a orientação espacial da pelve. A PT é um parâmetro posicional, assim como a SS. A possibilidade de rotação da pelve em torno 
do eixo das cabeças femorais é um dos melhores mecanismos de regulação do $\mathrm{SB}^{4}$

A perícia clínica e a literatura atual ${ }^{5}$ sugerem que o restabelecimento de um SB fisiológico é extremamente importante, e deve orientar as correções cirúrgicas. O entendimento dos parâmetros espinopélvicos e sua importância na biomecânica da coluna vertebral ganharam relevância, e vários $\operatorname{artigos}^{6}$ tentaram descrevê-los e sua relação, mas há dados limitados sobre a resposta dos parâmetros espinopélvicos e o alinhamento do plano sagital da coluna vertebral ao tratamento cirúrgico da SK. O objetivo deste estudo é avaliar e correlacionar os parâmetros pélvicos, o SB e o resultado funcional dos pacientes submetidos ao tratamento cirúrgico da SK.

\section{Material e Métodos}

Os registros de pacientes submetidos à correção de SK em nossa instituição entre janeiro de 2005 e dezembro de 2016 foram recuperados do nosso banco de dados. O diagnóstico foi baseado nos critérios radiológicos de Sorensen. ${ }^{3}$ Foram excluídos pacientes com deformidade congênita da coluna vertebral, doença neuromuscular ou sindrômica, cirurgia anterior da coluna vertebral, tumor na coluna vertebral, cifose postural ou deformidade primária da escoliose, espondilolistese, ou espondilólise. A indicação para cirurgia foi uma TK ou TLK com curva superior a $70^{\circ}$, e dor persistente nas costas e sem resposta satisfatória ao manejo conservador. Para ser incluído no estudo, todos os parâmetros do paciente tinham que estar disponíveis, e o acompanhamento tinha que ser concluído. Todos os pacientes foram submetidos a um procedimento de correção apenas posterior, com osteotomias periapicais e fixação de parafuso de pedículo pela mesma equipe cirúrgica, composta por dois cirurgiões seniores. O limite superior da instrumentação foi a vértebra Cobb da extremidade proximal, e a vértebra instrumentada inferior foi a vértebra estável sagital ou a primeira vértebra lordótica, de acordo com uma decisão tomada previamente. Durante a cirurgia, foram tomados cuidados para preservar os ligamentos supraespinais e interespinais, os processos espinhosos do limite superior da instrumentação, e o nível imediatamente acima dele. A deambulação sem aparelho é realizada no primeiro ou segundo dia após a cirurgia.

As medições radiográficas foram obtidas por meio de radiografias da coluna de longa duração antes da cirurgia e durante todo o seguimento. Em cada paciente, os parâmetros medidos foram: TK; lordose lombar (LL); SB; PI; PT; e SS. Todas as medições foram realizadas por dois médicos, e os valores médios foram registrados (- Fig. 1).

Outros parâmetros de imagem registrados foram 0 número de vértebras instrumentadas, o nível de fusão distal, e as complicações durante o período de seguimento, como a cifose juncional radiográfica, definida como a placa final caudal da vértebra instrumentada superior (UIV) à placa final cefálica de duas vértebras suprajacentes acima da UIV, e a mesma para a última vértebra inferior instrumentada. ${ }^{7}$

O questionário de 22 itens da Scoliosis Research Society (SRS-22), traduzido para nossa língua nativa, foi preenchido por todos os pacientes.

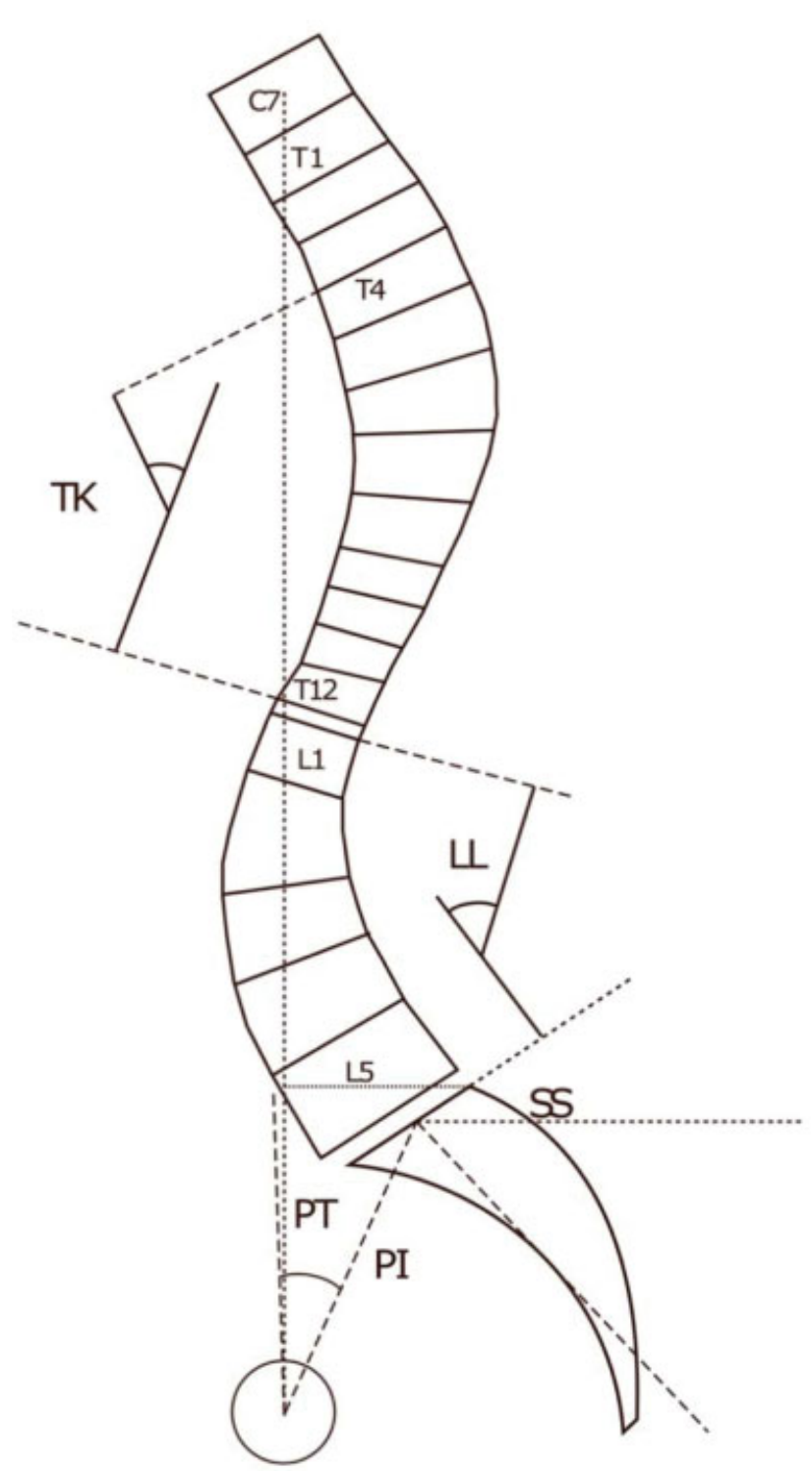

Fig. 1 Medições pré e pós-operatórias: cifose torácica (TK) - o ângulo entre a placa final superior de T4 até a placa final inferior de T12; lordose lombar (LL) - o ângulo entre a placa superior de L1 e a placa superior de S1; equilíbrio sagital - a distância entre a linha de prumo C7 e o canto posterossuperior de $\mathrm{S} 1$; incidência pélvica $(\mathrm{PI})$ - o ângulo entre a linha perpendicular à placa sacral em seu ponto médio e a linha que liga o ponto ao eixo médio das cabeças femorais; inclinação pélvica (PT) - o ângulo entre a linha que liga o ponto médio da placa sacral ao eixo médio das cabeças femorais e uma linha vertical; inclinação sacral (SS) - o ângulo entre a placa sacral e o plano horizontal.

Toda a análise estatística foi realizada utilizando-se o programa Statistical Package for the Social Sciences (SPSS, IBM Corp., Armonk, NY, EUA), versão 21.0 para Windows. O teste $t$ de Student foi utilizado para avaliar as diferenças entre os grupos nos valores médios das variáveis contínuas normalmente distribuídas. Comparações estatísticas entre os grupos pré-operatório e pós-operatório foram realizadas utilizando-se o teste U de Mann-Whitney. Foram utilizadas amostras emparelhadas em teste $t$ para a comparação de medições pré-operatórias e pós-operatórias. Valores de $p<0,05$ foram considerados estatisticamente significativos. 
Os autores obtiveram aprovação institucional do conselho de revisão para todos os aspectos deste estudo.

\section{Resultados}

Foram incluídos 19 pacientes ( 3 do sexo feminino, 16 do sexo masculino, com idade média de 18,4 anos na cirurgia) submetidos à cirurgia de correção de SK. O tempo de seguimento variou de 2 a 12 anos, com tempo médio de 6 anos. $O$ ápice da deformidade foi na coluna torácica, seguindo a distribuição: T7-6 casos; T8-9 casos; T9-4 casos. Em média, foram fundidos 11 níveis: o nível de fusão distal foi L1 em 3 casos, L2 em 10 casos, L3 em 5 casos, e L4 em 1 caso, e todos os pacientes submetidos a um procedimento somente posterior. A cifose pré-operatória média foi de $83^{\circ}$, com uma correção média para $57^{\circ}$. A lordose lombar média pré-operatória foi de $66^{\circ}$, com uma correção espontânea pós-operatória para $47^{\circ}$.

Quanto aos parâmetros pélvicos pré-operatórios, as médias da PI, da PT, e da SS foram de $48^{\circ}, 10^{\circ}$ e $39^{\circ}$, respectivamente, e, no pós-operatório esses valores foram de $50^{\circ}, 16^{\circ}$ e $35^{\circ}$, respectivamente. O SB médio pré-operatório avaliado pela distância do fio de prumo de $\mathrm{C} 7$ ao corpo vertebral S1 posterossuperior foi neutro (tendência negativa de $-8 \mathrm{~mm})$ e mantido após a correção cirúrgica $(-7 \mathrm{~mm})$ (-Fig. 2).

Quanto às complicações durante o período de seguimento, foram observadas três cifoses juncionais (duas proximais e uma distal) - duas necessitando de cirurgia de revisão, uma má consolidação, e uma deiscência de sutura. Os resultados funcionais avaliados por meio do questionário SRS-22 obtiveram uma pontuação média de 4,0. Relacionando a pontuação no SRS-22 com os parâmetros de imagem, verificou-se que o SB obtido no pós-operatório não teve influência no desfecho funcional $(p=0,125)$, nem na LL pósoperatória $(p=0.851)$. Embora tenha havido uma tendência a pontuações mais baixas no SRS-22 com o aumento da cifose pós-operatória, essa pontuação não afetou significativamente o resultado funcional $(p=0,079)$ ( - Tabela 1 ).

\section{Discussão}

O tratamento cirúrgico da SK não consiste apenas na correção da TK; consiste também em um desafio de equilíbrio por meio do alinhamento sagital.

Os parâmetros espinopélvicos sagitais foram avaliados em muitas áreas de deformidade espinhal, incluindo escoliose precoce, ${ }^{8}$ escolioses idiopáticas, ${ }^{9}$ e escoliose idiopática adolescente. ${ }^{10}$ No entanto, há uma escassez de literatura sobre o alinhamento espinopélvico sagital na SK.

O alinhamento espinopélvico sagital esteve ligado à qualidade de vida relacionada à saúde, principalmente porque o desequilíbrio sagital global e seus mecanismos compensatórios estavam associados ao aumento do consumo de energia, o que influencia negativamente a qualidade de vida. ${ }^{11,12}$

O aumento patológico da TK na SK leva a um desenvolvimento compensatório de uma hiperlordose lombar com o objetivo de alcançar um novo estado de equilíbrio.
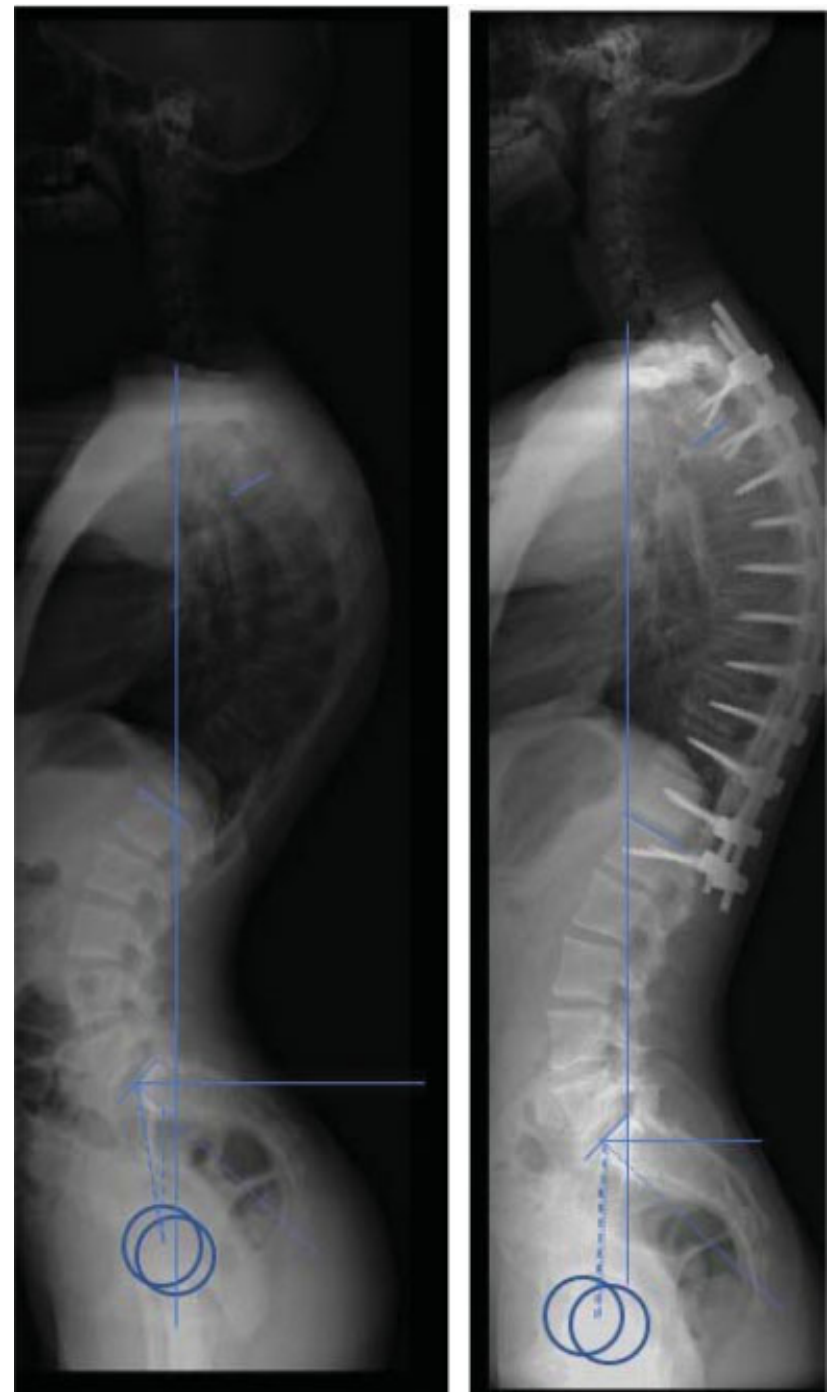

Fig. 2 Caso clínico - homem, 17 anos. Ele manteve o alinhamento sagital neutro. Melhora da cifose pós-operatória e da lordose lombar, de $80^{\circ}$ a $56^{\circ}$, e de $84^{\circ}$ a $55^{\circ}$, respectivamente.

Neste estudo, os valores médios da TK pré-operatória e da LL foram de $83^{\circ}$ e $66^{\circ}$, respectivamente. Após a correção cirúrgica da hipercifose, o valor médio da LL foi de $47^{\circ}$, por isso houve melhora espontânea da hiperlordose lombar em níveis não incluídos na fusão, conforme descrito na literatura ${ }^{13-17}$ e também verificado por Ashraf et al. ${ }^{16}$ em estudo retrospectivo de 18 pacientes submetidos ao tratamento cirúrgico.

Os valores médios pré-operatórios de PI, da PT e da SS foram de $48^{\circ}, 10^{\circ}$ e $39^{\circ}$, respectivamente. Estes são consistentes com os relatórios anteriores de Mac-Thiong et al., ${ }^{18} \mathrm{em}$ que os valores médios da PI, PT e SS foram de $49^{\circ}, 8^{\circ}$ e $41^{\circ}$, respecitvamente, para crianças e adolescentes normais. Essas observações são consistentes com o estudo publicado por Cahill et al., ${ }^{19}$ que avaliaram as diferenças nos parâmetros pélvicos entre pacientes com SK e controles normais não afetados, o que permitiu concluir que não houve diferença estatisticamente significativa nos parâmetros pélvicos. Portanto, isso sugere que a SK não é impulsionada por um problema inerente de anatomia pélvica que resulta em 
Tabela 1 Distribuição do equilíbrio sagital pós-operatório, resultados de cifose e lordose lombar e resultado funcional

\begin{tabular}{|l|l|l|l|l|}
\hline Caso & Equilíbrio sagital final & Cifose pós-operatória (graus) & Lordose lombar pós-operatória (graus) & SRS-22 \\
\hline 1 & Neutro & 42 & 27 & 3,5 \\
\hline 2 & Negativo & 65 & 22 & 3,7 \\
\hline 3 & Neutro & 52 & 47 & 4,12 \\
\hline 4 & Negativo & 51 & 37 & 4,05 \\
\hline 5 & Neutro & 59 & 47 & 3,27 \\
\hline 6 & Neutro & 67 & 45 & 4 \\
\hline 7 & Neutro & 67 & 21 & 4,63 \\
\hline 8 & Negativo & 30 & 23 & 4,5 \\
\hline 9 & Positivo & 69 & 68 & 4,2 \\
\hline 10 & Neutro & 41 & 47 & 3,8 \\
\hline 11 & Neutro & 74 & 56 & 4,05 \\
\hline 12 & Negativo & 63 & 55 & 4,5 \\
\hline 13 & Neutro & 56 & 62 & 4,27 \\
\hline 14 & Positivo & 51 & 49 & 4,45 \\
\hline 15 & Negativo & 67 & 45 & 4,1 \\
\hline 16 & Neutro & 69 & 68 & 3,2 \\
\hline 17 & Negativo & 49 & 64 & 4,4 \\
\hline 18 & Neutro & 60 & 53 & 4,8 \\
\hline 19 & Positivo & & 3,27 \\
\hline
\end{tabular}

Abreviatura: SRS-22, questionário de 22 itens da Scoliosis Research Society.

mecânica anormal na coluna torácica ou toracolombar com posterior encolhimento vertebral secundário, mas que as alterações anatômicas vistas no ápice da deformidade na SK são de fato o processo patológico primário. ${ }^{19}$

O SB pós-operatório é um importante preditor de resultados funcionais ao retratar as forças musculares que agem na coluna vertebral e a energia associada gasta. ${ }^{20}$ Neste estudo, os valores médios do SB permaneceram dentro da faixa normal antes e depois da cirurgia, que também foi descrita por Guler et al. ${ }^{21}$ após a correção cirúrgica da SK.

No período pós-operatório, os valores médios dos parâmetros pélvicos foram de $50^{\circ}$ para a $\mathrm{PI}, 16^{\circ}$ para PT, e $35^{\circ}$ para a SS. Embora a PI seja um parâmetro morfológico, e, portanto, não deve mudar, essa diferença é aceitável, e pode ser explicada pela subjetividade das medições nas radiografias. Em relação à PT, ela varia de $0^{\circ}$ a $25^{\circ}$, o que pode justificar o aumento dos valores pré-operatórios, que pode ser devido à inclusão de pacientes que tiveram algumas complicações durante o acompanhamento. Em última análise, no pós-operatório, os valores médios permaneceram dentro dos limites normais, o que permitiu manter a razão descrita da PI igual à soma da PT e da SS. ${ }^{4}$ Outras fórmulas tentam correlacionar os parâmetros sagital espinhal e pélvico na SK. Berderman et al.,"22 por exemplo, descreveram uma nova correlação entre parâmetros comoTKou TLK, PI e LL, e concluíram que $\left(\mathrm{TK}-45^{\circ}\right)+\left(\mathrm{TLK}-0^{\circ}\right)+(\mathrm{PI}-\mathrm{LL})$ mantido dentro da faixa de $\pm 10^{\circ}$ é uma fórmula valiosa para avaliar o SB global em pacientes com SK em esqueleto maduro.

O nível de fusão distal na SK é um tema controverso, e deve haver um equilíbrio entre minimizar o risco de desenvolver problemas de junção distal e preservar os níveis de movimento. ${ }^{23}$ Além da discussão usual entre estender a fusão à vértebra estável sagital ou a primeira vértebra lordótica, Zhu et al. ${ }^{24}$ sugerem que o tipo de curva da SK também deve ser considerado, afirmando que a biomecânica de cada curva é diferente. Esses autores ${ }^{24}$ sugerem ainda que, no tipo de curva toracolombar, deve-se utilizar uma fusão mais curta, ao passo que no tipo torácico, esta instrumentação deve ser estendida até a vértebra sagital estável. Neste estudo, apenas curvas torácicas foram incluídas, e o nível de fusão distal coincidiu principalmente com a vértebra sagital estável (15 versus 4 casos).

A ocorrência de complicações no tratamento cirúrgico da SK tem sido objeto de estudo de diversos trabalhos publicados, ${ }^{25}$ nos quais foi relatada uma taxa global de $14 \%$.

Além da escolha do nível da fusão, a prevenção do desenvolvimento da cifose juncional inclui, hoje em dia, o estudo dos parâmetros espinopélvicos, cujos resultados podem ser a causa desse desenvolvimento.

A cifose juncional apresenta incidências que podem chegar a $30 \%$, dependendo da população do estudo, ${ }^{26}$ e, embora a cifose juncional proximal (PJK) seja mais comum, a cifose juncional distal (DJK) é mais frequentemente implicada na cirurgia de revisão.

Ao relacionar o desenvolvimento da DJK com os parâmetros espinopélvicos, Ghasemi et al. ${ }^{27}$ sugeriram que há um risco aumentado de desenvolver DJK em pacientes mais jovens, e concluíram que pacientes com maior grau de correção da cifose torácica e com valores absolutos mais negativos do SB tiveram maior risco de desenvolver essa complicação. No entanto, no 
nosso caso de DJK, não observamos nenhuma dessas condições, pois o paciente tinha uma TK dentro dos valores médios préoperatórios $\left(80^{\circ}\right)$ e um SB normal (+ $\left.10 \mathrm{~mm}\right)$, e, em relação à sua idade no momento da correção cirúrgica, ele também estava próximo do valor médio (18 anos).

Em relação à PJK, Sardar et al. ${ }^{28}$ relatam que uma TK maior, tanto antes da cirurgia quanto no acompanhamento final, tende a estar associada ao desenvolvimento de PJK, e essa complicação também está diretamente correlacionada com a magnitude da PI. Em nossos dois casos de PJK, encontramos valores elevados de PI $\left(59^{\circ}\right.$ e $\left.60^{\circ}\right)$, o que está de acordo com descritos anteriormente.

A SK está associada a uma piora de qualidade de vida e a uma diminuição das pontuações no SRS-22 em todos os domínios quando comparada com outras deformidades da coluna vertebral, como a escoliose idiopática adolescente, conforme relatado por Lonner et al. ${ }^{29}$ Esse prejuízo pode ser explicado pelos mecanismos compensatórios, ocorridos enquanto tenta-se preservar um SB global adequado, que são consumidores de energia e, muitas vezes, dolorosos. ${ }^{11}$ Glassman et al. ${ }^{30}$ constataram que o SB positivo estava linearmente correlacionado com a gravidade da dor nas costas, e é a medida radiográfica mais frequentemente associada a desfechos adversos. Além disso, eles também afirmam que um resultado bem-sucedido no longo prazo após a correção da cirurgia de TK pode ser mais precisamente previsto pela restauração do alinhamento do plano sagital em vez de por meio de uma correção isolada da TK. A pontuação média dos pacientes incluídos neste estudo foi de 4 (o valor máximo é 5), o que que revelou um bom resultado funcional global. No entanto, ao tentar relacionar essas pontuações no SRS-22 com o SB, não foi estabelecida uma relação estatisticamente significativa, embora elas estivessem dentro da faixa normal. As pontuações no SRS-22 não foram influenciadas pela TK nem pela LL, embora relatemos uma tendência a pontuações menores no SRS-22 com maiores graus de TK pós-operatória.

Existem algumas limitações deste estudo. Primeiro, o pequeno número de sujeitos incluídos. Segundo, o caráter retrospectivo da análise feita. Em terceiro lugar, todos os procedimentos foram realizados no mesmo hospital e pela mesma equipe cirúrgica.

\section{Conclusão}

O tratamento cirúrgico da SK visa não apenas corrigir a deformidade, como também alcançar um equilíbrio global da coluna vertebral. Verificamos uma melhora espontânea da hiperlordose lombar em níveis não incluídos na fusão após a correção da TK. Embora os resultados funcionais pós-operatórios tenham sido globalmente elevados, não encontramos nenhuma relação estatisticamente significativa com a TK nem com a LL, embora tenhamos relatado uma tendência a pontuações menores no SRS-22 com maiores graus de TK pós-operatória. Complicações pós-operatórias como a cifose juncional estão relacionadas não apenas a fatores já conhecidos - como magnitude de correção de curvas e seleção de níveis de fusão -, como também a novos parâmetros, como o alinhamento do plano sagital. A PI alta está associada a maior complicação da PJK, e esses parâmetros pélvicos devem ser considerados no momento do tratamento cirúrgico, conforme concluímos. No entanto, mais estudos são necessários neste campo.

\section{Suporte Financeiro}

Não houve suporte financeiro de fontes públicas, comerciais, ou sem fins lucrativos.

Conflito de Interesses

Os autores declaram não haver conflito de interesses.

\section{Referências}

1 Wood KB, Melikian R, Villamil F. Adult Scheuermann kyphosis: evaluation, management, and new developments. J Am Acad Orthop Surg 2012;20(02):113-121

2 Damborg F, Engell V, Andersen M, Kyvik KO, Thomsen K. Prevalence, concordance, and heritability of Scheuermann kyphosis based on a study of twins. J Bone Joint Surg Am 2006;88(10): 2133-2136

3 Sorensen K. Scheuermann's Juvenile Kyphosis: Clinical Practice, Appearances, Radiography, Aetiology and Prognosis. Philadelphia: Lippincott Williams \& Wilkins; 2001

4 Le Huec J, Aunoble S, Philippe L, Nicolas P. Pelvic parameters: origin and significance. Eur Spine J 2011;20(Suppl 5):564-571

5 Lonner BS, Newton P, Betz R, et al. Operative management of Scheuermann's kyphosis in 78 patients: radiographic outcomes, complications, and technique. Spine (Phila Pa 1976) 2007;32(24): 2644-2652

6 Tyrakowski M, Mardjetko S, Siemionow K. Radiographic spinopelvic parameters in skeletally mature patients with Scheuermann disease. Spine (Phila Pa 1976) 2014;39(18):E1080-E1085

7 Kim YJ, Bridwell KH, Lenke LG, Glattes CR, Rhim S, Cheh G. Proximal junctional kyphosis in adult spinal deformity after segmental posterior spinal instrumentation and fusion: minimum five-year follow-up. Spine (Phila Pa 1976) 2008;33(20): 2179-2184

8 El-Hawary R, Sturm PF, Cahill PJ, et al. Sagittal spinopelvic parameters of young children with scoliosis. Spine Deform 2013;1(05):343-347

9 Roussouly P, Gollogly S, Berthonnaud E, Labelle H, Weidenbaum M. Sagittal alignment of the spine and pelvis in the presence of L5s1 isthmic lysis and low-grade spondylolisthesis. Spine (Phila Pa 1976) 2006;31(21):2484-2490

10 Tanguay F, Mac-Thiong JM, de Guise JA, Labelle H. Relation between the sagittal pelvic and lumbar spine geometries following surgical correction of adolescent idiopathic scoliosis. Eur Spine J 2007;16(04):531-536

11 Lafage V, Schwab F, Patel A, Hawkinson N, Farcy JP. Pelvic tilt and truncal inclination: two key radiographic parameters in the setting of adults with spinal deformity. Spine (Phila Pa 1976) 2009;34(17):E599-E606

12 Schwab F, Lafage V, Patel A, Farcy J-P. Sagittal plane considerations and the pelvis in the adult patient. Spine (Phila Pa 1976) 2009;34 (17):1828-1833

13 Hosman AJ, Langeloo DD, de Kleuver M, Anderson PG, Veth RP, Slot $\mathrm{GH}$. Analysis of the sagittal plane after surgical management for Scheuermann's disease: a view on overcorrection and the use of an anterior release. Spine (Phila Pa 1976) 2002;27(02):167-175

14 Poolman RW, Been HD, Ubags LH. Clinical outcome and radiographic results after operative treatment of Scheuermann's disease. Eur Spine J 2002;11(06):561-569

15 de Jonge T, Illés T, Bellyei A. Surgical correction of Scheuermann's kyphosis. Int Orthop 2001;25(02):70-73 
16 Ashraf A, Noelle Larson A, Polly DW, Ferski G, Guidera KJ, Mielke $\mathrm{CH}$. Change in Sagittal Plane Alignment Following Surgery for Scheuermann's Kyphosis. Spine Deform 2014;2(05):404-409

17 Lonner BS, Parent S, Shah SA, et al. Reciprocal Changes in Sagittal Alignment With Operative Treatment of Adolescent Scheuermann Kyphosis-Prospective Evaluation of 96 Patients. Spine Deform 2018;6(02):177-184

18 Mac-Thiong JM, Labelle H, Berthonnaud E, Betz RR, Roussouly P. Sagittal spinopelvic balance in normal children and adolescents. Eur Spine J 2007;16(02):227-234

19 Cahill PJ, Steiner CD, Dakwar E, et al;Harms Study Group. Sagittal spinopelvic parameters in Scheuermann's kyphosis: a preliminary study. Spine Deform 2015;3(03):267-271

20 Lamartina C, Berjano P. Classification of sagittal imbalance based on spinal alignment and compensatory mechanisms. Eur Spine J 2014;23(06):1177-1189

21 Guler O, Akgul T, Korkmaz M, et al. Postoperative changes in sacropelvic junction in short-segment angular kyphosis versus Scheuermann kyphosis. Eur Spine J 2017;26(03):928-936

22 Bederman SS, Farhan S, Hu X, et al. Sagittal Spinal and Pelvic Parameters in Patients With Scheuermann's Disease: A Preliminary Study. Int J Spine Surg 2019;13(06):536-543

23 Yanik HS, Ketenci IE, Coskun T, Ulusoy A, Erdem S. Selection of distal fusion level in posterior instrumentation and fusion of Scheuermann kyphosis: is fusion to sagittal stable vertebra necessary? Eur Spine J 2016;25(02):583-589
24 Zhu W, Sun X, Pan W, et al. Curve patterns deserve attention when determining the optimal distal fusion level in correction surgery for Scheuermann kyphosis. Spine J 2019;19(09): 1529-1539

25 Coe JD, Smith JS, Berven S, et al. Complications of spinal fusion for scheuermann kyphosis: a report of the scoliosis research society morbidity and mortality committee. Spine (Phila Pa 1976) 2010; 35(01):99-103

26 Denis F, Sun EC, Winter RB. Incidence and risk factors for proximal and distal junctional kyphosis following surgical treatment for Scheuermann kyphosis: minimum five-year follow-up. Spine (Phila Pa 1976) 2009;34(20):E729-E734

27 Ghasemi A, Stubig T, A Nasto L, Ahmed M, Mehdian H. Distal junctional kyphosis in patients with Scheuermann's disease: a retrospective radiographic analysis. Eur Spine J 2017;26(03): 913-920

28 Sardar ZM, Ames RJ, Lenke L. Scheuermann's Kyphosis: Diagnosis, Management, and Selecting Fusion Levels. J Am Acad Orthop Surg 2019;27(10):e462-e472

29 Lonner B, Yoo A, Terran JS, et al. Effect of spinal deformity on adolescent quality of life: comparison of operative scheuermann kyphosis, adolescent idiopathic scoliosis, and normal controls. Spine (Phila Pa 1976) 2013;38(12):1049-1055

30 Glassman SD, Bridwell K, Dimar JR, Horton W, Berven S, Schwab F. The impact of positive sagittal balance in adult spinal deformity. Spine (Phila Pa 1976) 2005;30(18):2024-2029 